\title{
Impact of community paramedic interventions on transfers from long term care to emergency departments: results of a systematic review
}

\author{
Abraham Munene $^{1}$ (1) $\cdot$ Dominic Alaazi ${ }^{2} \cdot$ Jane Mathew $^{1} \cdot$ Patrick McLane $^{3} \cdot$ Greta Cummings $^{2} \cdot$ Jayna Holroyd-Leduc $^{1}$ \\ Received: 25 August 2021 / Accepted: 17 September 2021 / Published online: 14 October 2021 \\ (c) Canadian Association of Emergency Physicians (CAEP)/ Association Canadienne de Médecine d'Urgence (ACMU) 2021, corrected publication 2021
}

Keywords Long term care $\cdot$ Community paramedicine $\cdot$ Emergency departments $\cdot$ Transfer

Dear Editor,

Long term care (LTC) facilities are essential living arrangements for many older adults living with frailty who require assistance with their daily needs and management of chronic health issues. In times of acute medical distress, LTC residents may require transfer to the emergency department (ED). Approximately $25 \%$ of Canadian LTC residents are transferred to the ED when experiencing acute changes in health status. While sometimes necessary, transfers from LTC to the ED can lead to poor outcomes for LTC residents and add strain on resources available in ED and ambulance transport services. Several common acute changes in health could be managed within LTC, if appropriate resources were available. However, many LTC facilities have high resident to staff ratios, overworked employees, a paucity of skilled healthcare workers, and limited access to resources needed to manage acute issues. Therefore, finding efficient, effective, and affordable ways to support LTC staff to care for acute issues within LTC are needed to improve both patient and health system outcomes.

Community paramedicine, a form of community-based health care delivery that utilizes specially trained emergency medical services (EMS) personnel and systems, has the potential to help meet the acute health needs of LTC residents. Results from our recent systematic review of five databases (Medline; CINAHL, PubMed, Embase, Cochrane)

Abraham Munene

abraham.munene2@ucalgary.ca

1 Cumming School of Medicine, University of Calgary (Foothills Campus), South Tower, 1403-29 Street NW T2N 4N1, Calgary, AB, Canada

2 Faculty of Nursing, University of Alberta, Edmonton, AB, Canada

3 Alberta Health Services, Calgary, AB, Canada searched from 1990 to June 2020 provided 19,178 citations. 181 articles were reviewed; four articles from one observational study (one Canadian study) suggest community paramedicine programs can reduce transfers from LTC to ED [1-4].

In the Canadian study, LTC residents were successfully treated by community paramedicine on-site and stayed at the LTC facility in nearly $70 \%$ of cases, compared to only $21 \%$ of those who were attended to by traditional EMS [1]. A low relapse rate (i.e., repeat calls $48 \mathrm{~h}$ after community paramedicine visit) was also reported in the study. Not surprisingly, higher call times were noted when community paramedicine attended to residents in LTC as compared to EMS [1].

Questions remain regarding the impact of community paramedicine programs on resident outcomes, the acuity levels of patients eligible for community paramedicine care, the acceptability of community paramedicine programs by both residents and health practitioners, training required for community paramedicine involved in LTC, and the cost effectiveness of having community paramedicine programs involved in the acute care of residents within LTC [5]. Going forward, we believe this is an area of research that requires further attention, given the projected impact of the aging Canadian population on EDs.

\section{Declarations}

Conflict of interest The authors declare no conflict of interest with this publication. Results of the systematic review are available upon request.

\section{References}

1. Jensen JL, Travers AH, Bardua DJ, Dobson T, Cox B, McVey $\mathrm{J}$, et al. Transport outcomes and dispatch determinants in a 
paramedic long-term care program: a pilot study. Can J Emerg Med. 2013;15(4):206-13.

2. Jensen JL, Travers AH, Marshall EG, Leadlay S, Carter AJE. Insights into the implementation and operation of a novel paramedic long-term care program. Prehosp Emerg Care. 2014;18(1):86-91.

3. Marshall EG, Clarke B, Peddle S, Jensen J. Care by design: new model of coordinated on-site primary and acute care in long-term care facilities. Can Fam Phys. 2015;61(3):e129-34.
4. Jensen JL, Marshall EG, Carter AJE, Boudreau M, Burge F, Travers $\mathrm{AH}$. Impact of a novel collaborative long-term care -ems model: a before-and-after cohort analysis of an extended care paramedic program. Prehosp Emerg Care. 2016;20(1):111-6.

5. van Vuuren J, Thomas B, Agarwal G, MacDermott S, Kinsman L, O'Meara P, et al. Reshaping healthcare delivery for elderly patients: the role of community paramedicine a systematic review. BMC Health Serv Res. 2021;21(1):29. 